\title{
Match Analysis in a University Taekwondo Championship
}

\author{
Coral Falco ${ }^{1}$, Raúl Landeo ${ }^{2}$, Cristina Menescardi ${ }^{1}$, José Luis Bermejo ${ }^{3}$, Isaac Estevan ${ }^{3}$ \\ ${ }^{1}$ Department of Physical Activity and Sport Sciences, Catholic University of Valencia, Valencia, Spain \\ ${ }^{2}$ School of Exercise Science, Australian Catholic University, Sydney, Australia \\ ${ }^{3}$ Department of Management and Applied Sciences, Catholic University of Valencia, Valencia, Spain \\ Email: coral.falco@ucv.es
}

Received December $5^{\text {th }}, 2011$; revised December $28^{\text {th }}, 2011$; accepted January $15^{\text {th }}, 2012$

\begin{abstract}
The aim of this study was to analyze the performance of medallists' taekwondo athletes in University level Championship. Kicking actions were grouped into three categories; circular, linear and with a previous spin kicks. Performance was evaluated based on 1) the type and height of the kicks employed, 2) the attacking and counterattacking nature of the kicking actions and 3) the scoring profiles of these. Forty-five matches from the four Olympic weight categories were notationally analyzed in male and female populations. Kruskal-Wallis tests revealed that the reading scores in the four weight categories differed significantly in males for linear kicks, and in circular kicks to the chest, attacking kicks and total actions ( $p<$ 0.05 ) for females. A greater number of circular kicks was recorded in male and female taekwondo athletes; this was followed by linear kicks and those with a previous spin. Kicks to the chest were also more common than kicks to the head as well as attacking kicks were used more frequently than counterattacking kicks. Given the new competition rules, coaches are encouraged to induce athletes to increase the frequency of kicks to the head and with a previous spin to maximise the point scoring rate per kicks delivered.
\end{abstract}

Keywords: Notational Analysis; Taekwondo; Attack; Counterattack; Kick

\section{Introduction}

Over the past few years, notational analysis research has developed many sport analysis systems to describe in detail the actions of athletes in competition (Liebermann, Katz, Hughes, Bartlett, McClements, \& Franks, 2002). This method of analyzing athletic skills is valuable in helping coaches improve their athletes' performance and increase the likelihood of success. Correct interpretation of notational analysis data allows coaches to provide appropriate feedback, motivate athletes, monitor improvements throughout the entire season (Byra \& Scott, 1983), and provide valuable insight into the athletes' strengths and weaknesses in order to allocate practice time effectively.

Notational analysis has been used extensively to study the tactical aspects of squash (Hong, Robinson, \& Chan, 1998; Hughes, 1993; Hughes \& Franks, 1993), football (Hughes, 1993), basketball (Miller \& Bartlett, 1994), rugby (Hughes \& Clarke, 1994), netball (Palmer, Hughes, \& Borrie, 1994) and badminton (Hong \& Tong, 2000). In taekwondo, notational analysis systems has traditionally been used to analyze injury rates during competition (see Lystad et al., 2008 for complete review), to observe physiological demands of the match (Bouhlel, Jouini, Gmada, Nefzi, Abdallah, \& Tabka, 2006; Butios \& Tasika, 2007) or to describe athletes' technical profiles (Kazemi, Waalen, Morgan, \& White, 2006). Limited research on different taekwondo styles championships (i.e., Matsuhigue, Hartmann, \& Franchini, 2009) was found while research on competitive taekwondo as general match, tactical (i.e., Santos, Franchini, \& Lima-Silva, 2011) or technical indicators (Luk, Hong, \& Chu, 2001) is lacking.

Taekwondo is a combat sport where male and female athletes compete in different weight divisions. Taekwondo matches are contested in three two minute rounds with one minute rest. Points are awarded when a successful kick makes contact with an allowed body region. The location of the kicks determines their scoring; one point for a valid attack on trunk protector, two points for a valid spin kick to the trunk protector, three points for a valid kick to the head and four points for a valid turning kick to the head. For example 4 penalty points results in a default of the athlete that scored the penalty points. When a gap of 12 points is reached at the time of the completion of 2 nd round and/or at any time during the 3rd round the match is stopped and the winner is declared (WTF, 2011).

Thus the kicking action is the most important component of a match. Kicking actions in Taekwondo can be classified as being: circular, targeting the lateral side of an opponent, linear aimed to the opponent's anterior end, and rotational which are performed with a previous spin (Serina \& Lieu, 1991). Understanding the various strategic approaches used in taekwondo competition as well as the physiological, psychological, technical and tactical demands of the sport is of importance to broaden the knowledge of coaches and to train athletes more effectively for competitive situations. The aim of this study was to analyze the number of kicking actions of male and female taekwondo athletes in the semi-final and final contests using the four Olympic weight divisions in a university level competition, with emphasis on the type of kick, and height of the kick, in both attacking and counterattacking actions as well as points obtained in the match.

\section{Method}

\section{Participants}

Sixty one, male $(\mathrm{n}=30)$ and female $(\mathrm{n}=31)$, taekwondo athletes aged from 18 to 33 years $(\bar{x}=22.26 ; S D=3.41)$ signed a consent form and participated in the study. Matches 
from the Spanish University Championship (May, 2011) were recorded. This competition counted towards the participation in the Universiade Championship, Shenzhen, China, 2011.

Following criteria by Matsushigue et al. (2009) and Santos et al. (2011), for the reporting of results the eight weight divisions were grouped into the four categories of the Olympic competition: Fin and Fly; Bantam and Feather; Light and Welter; and Middle and Heavy. A total of 45 matches from these groups: male: $\leq 58 \mathrm{~kg}$ ( 4 semifinals and 2 finals), $58-68 \mathrm{~kg}$ ( 3 semifinals and 2 finals), $68-80 \mathrm{~kg}$ (4 semifinals and 2 finals), and $\geq 80 \mathrm{~kg}$ ( 3 semifinals and 2 finals) and females: $\leq 49 \mathrm{~kg}$ ( 3 semifinals and 2 finals), 49 - $57 \mathrm{~kg}$ (4 semifinals and 2 finals), $57-67 \mathrm{~kg}$ ( 4 semifinals and 2 finals) and $\geq 72 \mathrm{~kg}$ ( 4 semifinals and 2 finals). Three semifinals, two in male (one from $58-68 \mathrm{~kg}$ and another one from $\geq 80 \mathrm{~kg}$ ) and one in female (one from $\leq 49 \mathrm{~kg}$ ), were not contested because of injuries.

\section{Procedures}

A total of nine kicking parameters were defined: total number of actions, type of action, type of kick, area of kick and punctuation. Type of action was defined as attacking or counterattacking. The movement of the athlete, who started the sequence, was defined as an attacking action. The movement of the athlete to defend against an opponent's attack was defined as a counterattacking action (Luk et al., 2001). Type of kick was categorized into three variables: linear (i.e., pushing), circular (i.e., roundhouse) and with a previous spin (i.e., back) (Serina \& Lieu, 1991). The section of kicking was classified into chest and head, which are the only scoring areas in Taekwondo. The official videotapes from all the matches at the Spanish University Championship in 2011 were recorded onto DVD and analyzed using the Kinovea Software. Following standard procedures in combat sports analysis (Kazemi, Waalen, Morgan, \& White, 2006; Matsuhigue, Hartmann, \& Franchini, 2009; Salvador, Suay, Martinez-Sanchis, Simon, \& Brain, 1999; Santos et al., 2009), a trained investigator analyzed all videos to determine the pre-defined parameters. Previous research employing the same analysis reported reliable measures with an intraclass correlation coefficient (ICC) of 0.93 (Salvador et al., 1999). From a total of 5 randomly selected rounds an ICC $=$ 0.85 was obtained, revealing that the analysis conducted in this study was highly reliable for all variables investigated, with values ranging from 0.79 to $0.89(p<0.01)$.

\section{Statistical Analysis}

Using a Kolmogorov-Smirnov test data were found not normally distributed, therefore non-parametrical tests, i.e. KruskalWallis test followed by a Mann-Whitney U test, were used to assess the nine variable differences between weight divisions. The level of significance was set at $p<0.05$.

\section{Results}

The results of this study in the four weight categories revealed significant differences in males for linear kicks $\left[\chi^{2}(3)=\right.$ $8.57, p=0.04]$ and in females for circular kicks $\left[\chi^{2}(3)=22.57\right.$, $p=0.01]$, kicks to the chest $\left[\chi^{2}(3)=14.99, p=0.01\right]$, attacking kicks $\left[\chi^{2}(3)=10.62, p=0.01\right]$ and total actions $\left[\chi^{2}(3)=14.23\right.$, $p=0.01]$ in the match.

Linear kicks were performed significantly less by male flyweight compared to heavyweight $(\mathrm{U}=18.0, p=0.01)$, and to welterweight $(\mathrm{U}=18.0, p=0.02)$ divisions. Results are presented in Table 1.

The number of attacking kicks performed by female heavyweight athletes was significantly higher than that of featherweight $(\mathrm{U}=21.5, p=0.01)$ and flyweight $(\mathrm{U}=29.5, p=0.04)$ divisions, while the number of attacking kicks of featherweight was significantly higher than that of welterweight $(\mathrm{U}=36.5, p$ $=0.04)$. Athletes of the female heavyweight division used significantly more circular kicks than athletes of the welterweight $(\mathrm{U}=26.5, p=0.01)$, featherweight $(\mathrm{U}=1.0, p=0.01)$ and flyweight $(\mathrm{U}=8.5, p=0.01)$ divisions, while the number of circular kicks executed in the welterweight category was significantly higher than that in the featherweight $(\mathrm{U}=31.0, p=$ 0.02 ). The occurrence of kicks to the trunk of heavyweight was significantly higher than that in the welterweight $(\mathrm{U}=31.5, p=$ 0.02 ), featherweight $(\mathrm{U}=15.5, p=0.01)$, and flyweight $(\mathrm{U}=$ $14.5, p=0.01)$ divisions. There were in total significantly more kicks executed by athletes of the heavyweight division compared to the welterweight $(U=37.0, p=0.04)$, featherweight $(\mathrm{U}=13.5, p=0.01)$ and flyweight $(\mathrm{U}=19.5, p=0.01)$. These results are presented in Table 2.

Table 1.

Descriptive Mean $(\bar{x})$ and Standard Deviation (SD) of the male taekwondo athletes: total of actions, type of action (attacking or counterattacking), area of kick (chest or head), type of kick (circular, lineal or spin) and obtained points.

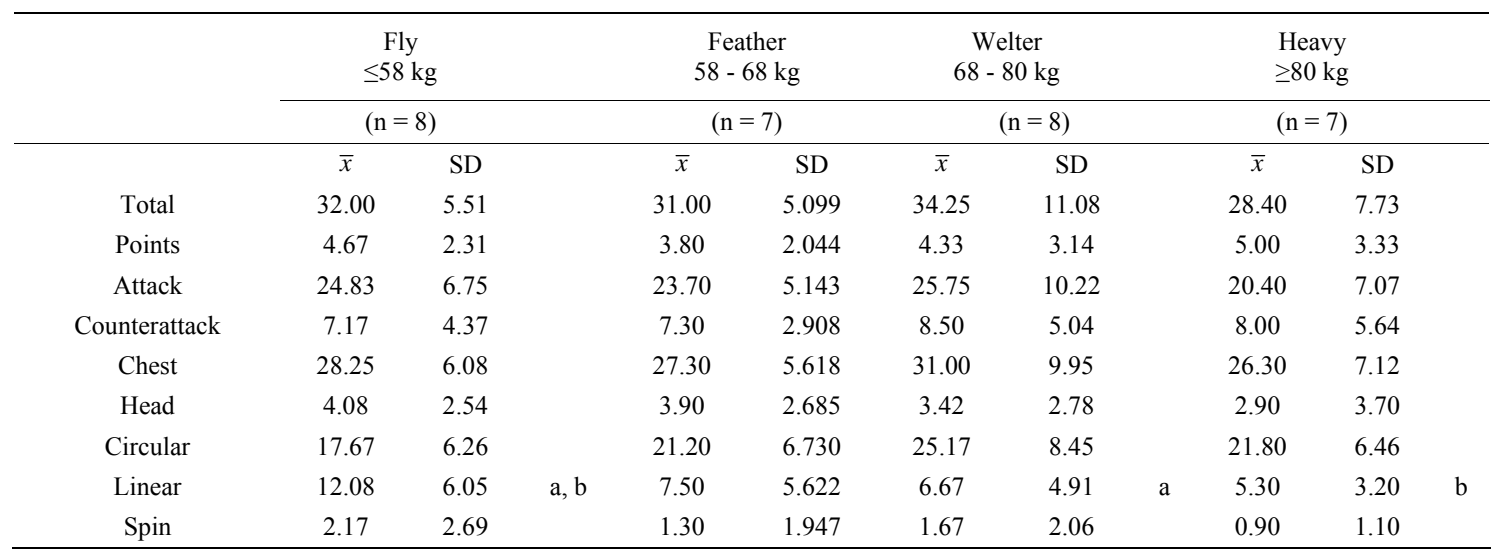

Note: The letters "a" and " $b$ " show differences between weight categories for each of the notational variables of the study $(p<0.05)$. 
Table 2.

Descriptive Mean $(\bar{x})$ and Standard Deviation (SD) of the female taekwondo athletes: total of actions, type of action (attacking or counterattacking), area of kick (chest or head), type of kick (circular, lineal or spin) and obtained points.

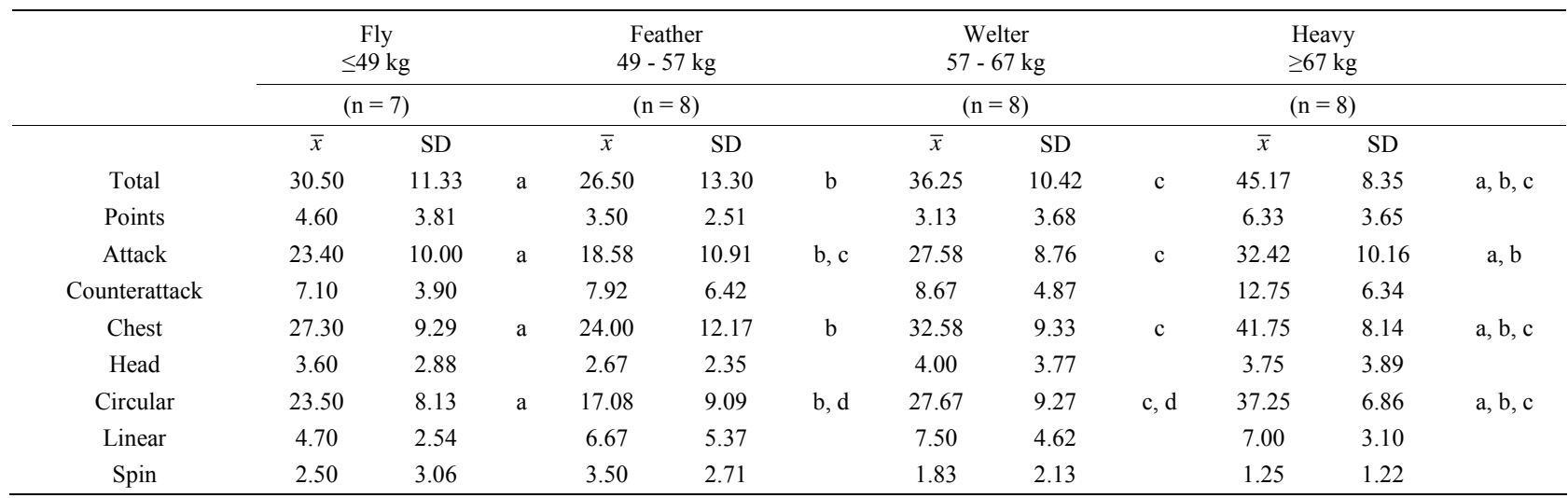

Note: The letters "a", "b" and "c" show differences between weight categories for each of the notational variables of the study $(p<0.05)$.

\section{Discussion}

The purpose of the present study was to analyze the performance of medallists' taekwondo athletes in a University Championship among the four Olympic weight divisions in semi-final and final contests. The performance indicators have been analyzed for male and female taekwondo athletes according to the total of actions, type of action, type of kick, area of kick as well as points obtained in the match. To that end, 45 taekwondo matches were analyzed. On average male taekwondo athletes performed thirty actions meanwhile obtained four points per match. This suggests that the efficiency of the kicks in terms of scoring points is rather low. Moreover, the number of total actions performed (attacking and counterattacking) is higher than what was found in previous studies. For example, Santos et al. (2011) reported that the flyweight division performed in average 8.6 kicks $(\mathrm{SD}=2.7)$, feather performed 6.6 kicks $(\mathrm{SD}=1.9)$, welterweight 7.5 kicks $(\mathrm{SD}=2.7)$, while heavyweight performed 6.8 kicks $(\mathrm{SD}=3.1$ ) per match, meanwhile Matsuhique et al. (2009) reported that the number of kicks per athlete per match was found to be 27 (SD = 13). This discrepancy in findings can be attributed to lower tactical awareness and reflexive behaviour in university level athletes from the present study compared to athletes in open competitions.

From the total actions performed, and in line with previous studies (Matsuhique et al., 2009; Santos et al., 2011), male taekwondo athletes used more attacking than counterattacking kicks in order to win the match. This suggests that to win the match, the performance of the taekwondo athletes must be motivated by attacking rather than counterattacking. However, results from the present study cannot be related to how points were obtained from specific kicks. Future studies should identify which types of kicks are used to obtain points and further address whether the attacking performance is motivated by the macth condition; winning or loosing a match.

It was previously also reported that male athletes from heavier weight divisions show typically slower fighting rhythm than lighter competitors (Butios \& Tasika, 2007). This was attributed to the higher energy expenditure of heavier athletes compared to lighter weight divisions (Santos et al., 2011) during a fighting bout. The available evidence however is indicative of homogeneity in performance between all male weight divisions. No differences in movement patterns and choice of kicking actions between heavier and lighter divisions were found, with the exception of the use of linear kicks. The use of linear kicks, in the flyweight division, was significantly higher than that of heavyweight and welterweight. Although no data on the power profiles of these kicks are available in this study it may be suggested that the power posed by linear kicks suffice to score points in lower weight divisions.

Despite of the action performed; attacking or counterattacking, and in line with previous studies (Luk et al., 2001; Matsuhique et al., 2009), it was also found that male taekwondo athletes prefer to use circular kicks followed by linear kicks and kicks with an initial spin. Research on kicks to the chest (Serina \& Lieu, 1991) has determined that circular kicks are faster than linear kicks, with the roundhouse kick being identified as the fastest kicking technique of all (Pieter \& Pieter, 1995) which may explain the preference for using circular kicks in competition.

Data from this study also revealed that taekwondo athletes preferred to use kicks to the chest more than to the head. That is, while athletes are more inclined to target the chest ahead of the head while performing a circular kick, kicks delivered to the head may pose higher injury risks for the receiving athletes. The concussion rate in taekwondo matches arising from roundhouse kicks (Koh et al., 2004) may partially be explained by this preference. No data on the fighting conditions that facilitate the execution of circular kicks are available in this study. It seems however that there are no timing differences in execution of a roundhouse kick to the chest or the head (i.e., Estevan, MolinaGarcia, Falco, \& Alvarez, 2010; Falco, Estevan, Menescardi, \& Ruiz, 2011; O’Sullivan et al., 2009) or the timing of between roundhouse kicks and kicks with a previous spin (i.e., Falco, Estevan, \& Vieten, 2011). Factors such as athlete position within the fighting area and relative to the opponent, scoring situation and their influences on triggering the execution of a given kick are yet to be explored. In light of the preference for lower kicks found in this study and given that the new competition rules reward kicks to the head and kicks initiated by a spinning motion, coaches are encouraged to guide athletes to increase the frequency of kicks directed to the head and those with an initial spin to maximise the action to scoring ratio.

The review of the current literature suggests that this is the first study that analyzes female taekwondo athletes; therefore, data on female athletes cannot be compared to other studies. It 
can be observed that female taekwondo athletes also used more attacking than counterattacking kicks and further more circular than linear kicks or kicks with an initial spin. Moreover, kicks to the chest were more common than kicks to the head. However, the four weight categories differed significantly from each other in; circular kicks, kicks to the chest, attacking kicks and total kicks performed in the match. That is, the female heavyweight category also preformed a higher number of actions compared to their lighter weight counterparts. Moreover, the heavy and welterweight categories developed a higher number of attacking kicks than their counterparts of the featherweight category. Similarly, the welterweight category performed a higher number of attacking kicks than their counterparts of featherweight category. The heavier weight divisions performed higher number of circular kicks, kicks to the chest, than their lighter counterparts. This is in contrast to what was observed in the male divisions, where athletes' behaviour and choice of kicking techniques seem to be independent of weight categories.

These data reveal that the technical and tactical characteristics of male and female competition are vastly different. These findings implicate that male and female competitors should train differently, at least during the tactical phase of training. On the other hand it may also be suggested that female competition is to some extent predictable, as the choice of kicking actions was observed to be highly dependent on weight division. This level of predictability, from the tactical point of view represents a disadvantage as the opponent can capitalize on that information. It is suggested that coaches of female competitors work on equipping their athletes with greater kicking repertoire to reduce the predictability of their actions.

Although in many university level competition world class as well as Olympic level athletes are competing, gathering data from a Spanish University level competition represent a limitation to the study. Limiting the population to those competing at university level, limits the generalizability of the study. Another limitation of the study is that player displacements were not analyzed, nor the match conditions when a particular kick was executed. It is still believed that these data can provide an insight into the tactical traits of Taekwondo matches and constitute a basis for future research.

In conclusion, male and female taekwondo athletes used more circular kicks, followed by linear and with a previous spin, and kicks to the chest were more used than kicks to the head as well as attacking kicks were used more than counterattacking kicks. It may be that in international high-level taekwondo competitions and national contests (Heller et al., 1998; Santos et al., 2011), effort is often interrupted by low-intensity movements or inactivity. Therefore, athletes in these competitions spend more time studying, approaching, and preparing a new attack on their opponents than on executing attacks. More research is warranted to better understand the dynamics of taekwondo matches across different countries as well as within different levels of competition. Also, it is of importance to study the type of attacking and counterattacking actions performed in order to know the quality of the kicks developed and how they relate to the tactical consideration based on when in the match they are performed.

\section{Acknowledgements}

This research was supported by the Catholic University of
Valencia (2011-007-002).

\section{REFERENCES}

Bouhlel, E., Jouini, A., Gmada, N., Nefzi, A., Abdallah, K. B., \& Tabka, Z. (2006). Heart rate and blood lactate responses during taekwondo training and competition. Science Sports, 21, 285-290. doi:10.1016/j.scispo.2006.08.003

Butios, S., \& Tasika, N. (2007). Changes in heart rate and blood lactate concentration as intensity parameters during simulated taekwondo competition. Journal of Sports Medicine and Physical Fitness, 47, 179-185.

Byra, M., \& Scott, A. (1983). A method for recording team statistics in volleyball. Volleyball Technical Journal, 7, 39-44.

Estevan, I., Molina-Garcia, J., Falco, C., \& Alvarez, O. (2010). Comparación de la eficiencia de la patada circular al pecho y a la cara en taekwondo, según la distancia de ejecución (Comparison of efficiency between the roundhouse kick to the chest and to the head according to the execution distance) [Spanish]. Revista Internacional de Ciencias del Deporte (International Journal of Sport Science), 21, 269-279.

Falco, C., Estevan, I., Menescardi, C., \& Ruiz, L. (2011). The roundhouse Kick in function of the two target level. Scientific Congress on Martial Arts and Combat Sports. In A. A. Figueiredo, \& C. Gutiérrez-García (Eds.), Associacao para o desenvolvimiento e investigacao de Viseu (pp. 36-38). Viseu, Portugal.

Falco, C., Estevan, I., \& Vieten, M. (2011). Kinematical analysis of five different kicks in taekwondo. Portuguese Journal of Sport Sciences, 11, 219-222.

Heller, J., Peric, T., Dlouha, R., Kohlikova, E., Melichna, J., \& Novakova, H. (1998). Physiological profiles of male and female taekwondo (ITF) black belts. Journal of Sports Science, 16, 243-249. doi: $10.1080 / 026404198366768$

Kazemi, M., Waalen, J., Morgan, C., \& White, A. R. (2006). A profile of Olympic taekwondo competitors. Journal of Sports Science \& Medicine, 5, 114-121.

Koh, J. O., Watkinson, E. J., \& Yoon, Y. J. (2004). Video analysis of head blows leading to concussion in competition taekwondo. Brain Injury, 18, 1287-1296. doi:10.1080/02699050410001719907

Liebermann, D. G., Katz, L., Hughes, M. D., Bartlett, R. M., McClements, J., \& Franks, I. M. (2002). Advances in the application of information technology to sport performance. Journal of Sports Sciences, 20, 755-769. doi:10.1080/026404102320675611

Luk, T. C., Hong, Y., \& Chu, D. P. K. (2001). Analysis of strategy used in taekwondo competition.

Matsushigue, K. A., Hartmann, K., \& Franchini, E. (2009). taekwondo: Physiological responses and match analysis. Journal of Strength and Conditioning Research, 23, 1112-1117. doi:10.1519/JSC.0b013e3181a3c597

Pieter, F., \& Pieter, W. (1995). Speed and force in selected taekwondo techniques. Biology of Sport, 12, 257-266.

Salvador, A., Suay, F., Martinez-Sanchis, S., Simon, V. M., \& Brain, P. F. (1999). Correlating testosterone and fighting in male participants in judo contests. Physiological Behaviour, 68, 205-209. doi:10.1016/S0031-9384(99)00168-7

Santos, V. G. F., Franchini, E., \& Lima-Silva, A. E. (2011). Relationship between attack and skipping in taekwondo contests. Journal of Strength and Conditioning Research, 25, 1743-1751. doi:10.1519/JSC.0b013e3181ddfb0f

Serina, E. R., \& Lieu, O. K. (1991). Thoracic injury potential of basic competition taekwondo kicks. Journal of Biomechanics, 24, 951- 960. doi:10.1016/0021-9290(91)90173-K

World taekwondo Federation (2011). Competition rules. URL (last checked 31 October 2011).

www.wtf.org/wtf_eng/site/rules/competition.html 